\title{
Scalar imaging velocimetry measurements of the velocity gradient tensor field in turbulent flows. I. Assessment of errors
}

\author{
Lester K. Su and Werner J. A. Dahm ${ }^{\text {a) }}$ \\ Gas Dynamics Laboratories, Department of Aerospace Engineering, The University of Michigan, \\ Ann Arbor, Michigan 48109-2118
}

(Received 25 January 1994; accepted 22 March 1996)

\begin{abstract}
The concept of flow field velocimetry based on scalar imaging measurements [Phys. Fluids A 4, 2191 (1992)] is here formulated in terms of an integral minimization implementation, where the velocity field $\mathbf{u}(\mathbf{x}, t)$ is found by minimizing weighted residuals of the conserved scalar transport equation, along with the continuity condition and a smoothness condition. We apply this technique to direct numerical simulation (DNS) data for the limiting case of turbulent mixing of a $\mathrm{Sc}=1$ passive scalar field. The spatial velocity fields $\mathbf{u}(\mathbf{x}, t)$ thus obtained demonstrate good correlation with the exact DNS fields, as do the statistics of the velocity and the velocity gradient fields. The results from this integral minimization implementation also show significant improvement over those from the direct inversion technique reported earlier. These results are shown to be largely insensitive to noise at levels characteristic of current fully resolved scalar field measurements. (C) 1996 American Institute of Physics. [S1070-6631(96)01507-3]
\end{abstract}

\section{INTRODUCTION}

Information on the fully resolved, three-dimensional spatial structure and simultaneous temporal dynamics of the full nine-component velocity gradient tensor field $\nabla \mathbf{u}(\mathbf{x}, t)$ at the small scales of turbulent flows is key to the development of physical models for these scales of turbulence. These scales are generally presumed to be quasi-universal in high Reynolds number flows, and are thus studied in a generic context. To date, this has been done almost exclusively via direct numerical simulations (DNS) of the Navier-Stokes equations. Laboratory experiments capable of directly yielding useful information on the detailed structure and dynamics of these scales have been comparatively few. Most have been limited to single-point measurements of a small subset of the full velocity gradient tensor field.

The earliest and still the most widely used experimental techniques for measuring one or several of the velocity gradient tensor components rely on multiple hot-wire or hotfilm probes. The original four hot-wire probe was developed in 1950 by Kovasznay ${ }^{1}$ for measurements of the streamwise component $\omega_{1}$ of the vorticity vector at a single spatial point. Corrsin and Kistler, ${ }^{2}$ and later Willmarth and $\mathrm{Lu},{ }^{3}$ Willmarth and Bogar, ${ }^{4}$ and Kastrinakis et al. $^{5-7}$ used probes of this type for streamwise vorticity component measurements in turbulent boundary layers. Vukoslavčević and Wallace ${ }^{8}$ subsequently developed an improved Kovasznay-type probe which used four independent wires to determine the streamwise vorticity component. Foss, ${ }^{9}$ Foss et al. ${ }^{10,11}$ and Haw, Foss and Foss ${ }^{12}$ have successfully used a similar four hotwire arrangement to measure the cross-stream component $\omega_{3}$ of the vorticity vector at a single point. A five-sensor hotfilm probe for simultaneously measuring the spanwise and normal vorticity components, $\omega_{2}$ and $\omega_{3}$, was developed and used by Eckelmann et al. ${ }^{13}$ Surveys of some of the characteristics of these vorticity probes and the data obtained from

${ }^{\text {a)} C o r r e s p o n d i n g ~ a u t h o r . ~ E l e c t r o n i c ~ m a i l: ~ w d a h m @ e n g i n . u m i c h . e d u ~}$ them are given by Wallace, ${ }^{14}$ Foss and Wallace, ${ }^{15}$ and Wallace and Foss. ${ }^{16}$ Kim and Fiedler ${ }^{17}$ subsequently used a sixsensor probe for simultaneous measurement of the streamwise and cross-stream vorticity components, $\omega_{1}$ and $\omega_{3}$. Recently Vukoslavčević, Wallace and Balint, ${ }^{18}$ and Balint, Wallace, and Vukoslavčevic ${ }^{19}$ have succeeded in building and using a nine-sensor hot-wire probe that yields simultaneous measurements for all three vorticity components $\omega_{1}$, $\omega_{2}$, and $\omega_{3}$. This probe permits resolution of the vorticity vector at a scale approximately six times coarser than the local Kolmogorov scale at their measurement location in a thickened turbulent boundary layer. Kit et al. ${ }^{20,21}$ have developed a nine-wire probe to measure components of the velocity gradient tensor, and more recently Tsinober, Kit, and Dracos $^{22}$ have assembled a 12-sensor hot-wire probe, and even a 20 -sensor probe without any common prongs, for measuring all nine independent components of the velocity gradient tensor at a single spatial point. In their grid turbulence experiment, this probe allowed resolution of length scales roughly five times coarser than the local Kolmogorov scale.

Among the strengths of these multiple hot-wire/film probe techniques are the ease with which extensive statistical samples can be collected, and their ability to isolate largescale contributions to the velocity and velocity gradient statistics. Despite the successes in development of these techniques, however, all have certain common characteristics that ultimately influence their ability to provide data on the structure and dynamics of the velocity gradient tensor field. Even in principle these methods allow time series measurements at only a single spatial "point," with one-dimensional spatial structure in the velocity gradient component fields discernible only if a Taylor hypothesis is accepted. Of a more practical nature, interference between the various wires and prongs in such probes leads to complex calibrations and the potential for erroneous measurements (e.g., see Vukoslavčević, Wallace, and Balint, ${ }^{18}$ and Tsinober, Kit, and $\left.\operatorname{Dracos}^{22}\right)$. 
In part to overcome these concerns about intrusive probes, more contemporary non-invasive optical techniques have been developed, of which laser Doppler velocimetry (LDV) remains by far the most popular. Lang ${ }^{23}$ developed an LDV with four focal volumes to measure the spanwise vorticity in a turbulent shear layer. More recent advances in laser diagnostics and high-speed data acquisition and processing capabilities have facilitated a variety of optically based, non-intrusive velocimetry methods which provide information over two-dimensional fields of many points. Reviews of some of these are given, for example, by Adrian, ${ }^{24,25}$ Merzkirch, ${ }^{26}$ Lauterborn and Vogel, ${ }^{27}$ Hesselink, ${ }^{28}$ Miles and Nosenchuck, ${ }^{29}$ Gad-el-Hak, ${ }^{30}$ and Chiang and Reid. ${ }^{31}$ The majority of these techniques involve particle imaging methods. These generally yield twocomponent velocity vectors over two-dimensional fields, though three-dimensional particle tracking (Nishino, Kasagi, and Hirata, ${ }^{32}$ Papantoniou and Maas ${ }^{33}$ ) and holographic particle image velocimetry (HPIV) (Haussmann and Lauterborn, ${ }^{34}$ Malyak and Thompson, ${ }^{35}$ Meng and Hussain, ${ }^{36}$ Scherer and Bernal $^{37}$ ) are being developed for measuring full, three-component vector velocity fields in complex flows. However, in all these particle based techniques, the seeding densities required to resolve the smallest spatial scales in a flow can become so high as to prevent sufficient optical penetration into the interior of the flow. Various techniques (Chu et al., ${ }^{38}$ Falco and Chu, ${ }^{39}$ Hassa, Paul and Hanson, ${ }^{40}$ Miles et al., ${ }^{41,42}$ Pearlstein and Carpenter, ${ }^{43}$ Gruen, ${ }^{44}$ Maas, ${ }^{45}$ Tokumaru and Dimotakis ${ }^{46}$ ) have also been proposed for extracting the velocity or certain components of the velocity gradient tensor field from scalar field measurements obtained through laser-induced fluorescence, phosphorescence, or other optical techniques.

The optical penetration difficulty can be circumvented by using an effectively continuous distribution of laser fluorescent dye molecules (or other suitable tracer) as the seed, whose size eliminates the Mie scattering associated with particulates and thus maintains optical transparency in the flow field. Instead of relying on the displacement of discrete particles seeded in the flow, we use the continuum field created by the concentration of a dynamically passive, conserved scalar quantity which is transported by the underlying turbulent flow (Dahm, Su, and Southerland ${ }^{47,48}$ ). This continuum field approach circumvents many of the difficulties noted above in conjunction with particle based methods, and is based on the recently demonstrated experimental capability for obtaining fully resolved, four-dimensional measurements of $\mathrm{Sc} \gg 1$ conserved scalar fields $\zeta(\mathbf{x}, t)$ in turbulent flows (Southerland and Dahm ${ }^{49-51}$ ). In scalar imaging velocimetry, extraction of the underlying space- and time-varying velocity field $\mathbf{u}(\mathbf{x}, t)$ from such scalar field measurements is based on the exact conserved scalar transport equation, which in dimensionless form is

$$
\left[\frac{\partial}{\partial t}+\mathbf{u} \cdot \boldsymbol{\nabla}-\frac{1}{\operatorname{ReSc}} \nabla^{2}\right] \zeta(\mathbf{x}, t)=0 .
$$

(All variables are taken to be normalized with reference length and velocity scales $l^{*}$ and $u^{*}$, and a reference scalar concentration value $\zeta^{*}$. The dimensionless scalar diffusivity involves the product of the Reynolds number $\operatorname{Re} \equiv u^{*} l^{*} / \nu$ and the Schmidt number $\mathrm{Sc} \equiv \nu / D$, where $\nu$ and $D$ are respectively the vorticity and scalar diffusivities.) Given fully space- and time-differentiable scalar field data, the only unknowns in this equation are the components of the velocity field $\mathbf{u}(\mathbf{x}, t)$. We have proposed ${ }^{47,48}$ that from sufficiently resolved, four-dimensional measurements of $\zeta(\mathbf{x}, t)$ and an appropriate set of additional constraints, one can extract the velocity field $\mathbf{u}(\mathbf{x}, t)$ despite that only the component of $\mathbf{u}(\mathbf{x}, t)$ along the scalar gradient vector $\nabla \zeta(\mathbf{x}, t)$ direction affects the scalar field evolution. The latter observation recognizes that, in addition to the true velocity field $\mathbf{u}(\mathbf{x}, t)$, Eq. (1) in principle admits all other velocity fields with streamlines confined to isoscalar surfaces. We assert, however, that in turbulent flows these spurious velocity fields can be excluded, since $\mathrm{Sc}>1$ scalar fields contain length scales smaller than those admissible in the underlying velocity fields. It will be seen that by taking into account the relative smoothness between the scalar and velocity fields in turbulent flows, it is indeed possible to determine the velocity field accurately.

There are two closely related methods by which this can be done. The first, referred to as direct inversion scalar imaging velocimetry, involves inverting the scalar transport equation (1) directly throughout the data space to yield the velocity component field $u_{\|}(\mathbf{x}, t)$ which lies along the local scalar gradient vector $\boldsymbol{\nabla} \zeta(\mathbf{x}, t)$. The full vector velocity field $\mathbf{u}(\mathbf{x}, t)$ is then extracted using the gradient field $\nabla u_{\|}(\mathbf{x}, t)$, recognizing that certain constraints must be met which reflect the relative smoothness between the scalar field data and the velocity field in turbulent flows. The second method, referred to as integral minimization scalar imaging velocimetry, is a refinement of the first that addresses the issues of relative smoothness and scalar field noise through a variational formulation. This method is still based on the scalar transport equation (1), but recognizes that any measurement errors in the scalar field data $\zeta(\mathbf{x}, t)$ will lead to a non-zero right-hand side in Eq. (1). The method thus determines the velocity field $\mathbf{u}(\mathbf{x}, t)$ which minimizes an integral composed of various residual terms. One of these residual terms measures the agreement of the velocity field and measured scalar field with the scalar transport equation (1), while another imposes the smoothness constraint as a regularization parameter. The right-hand side of Eq. (1) thus is not required to be exactly zero, but rather is minimized subject to smoothness and other constraints. By selecting the weight assigned to the smoothness constraint, the correct ratio of length scales in the velocity and scalar fields can be ensured. We will see that this integral minimization formulation has advantages over the direct inversion scheme, both in flexibility of application and in the potential accuracy of the results. Validation tests also show that the integral minimization formulation is capable of determining velocity and velocity gradient fields accurately in turbulent flows, even in the limit of unity Schmidt number where the finest length scales of the scalar gradient and velocity fields become comparable. A companion paper $(\mathrm{Su}$ and $\mathrm{Dahm}^{52}$ ), hereafter referred to as Part II, applies this technique to fully resolved, four-dimensional $\mathrm{Sc} \gg 1$ scalar field data to obtain the first non-invasive laboratory measurements for the full nine-component velocity gradient tensor 
field $\nabla \mathbf{u}(\mathbf{x}, t)$ and the pressure gradient field $\nabla p(\mathbf{x}, t)$ in a turbulent flow.

The direct inversion approach ${ }^{47,48}$ begins by recognizing that the convective term $\mathbf{u} \cdot \boldsymbol{\nabla} \zeta$ in the scalar transport equation (1) may be written as $u_{\|}|\nabla \xi|$, where $u_{\|}$is the projection of the velocity $\mathbf{u}$ onto the local scalar gradient vector direction, or

$$
u_{\|}(\mathbf{x}, t) \equiv \mathbf{u}(\mathbf{x}, t) \cdot \hat{e}_{\nabla \zeta}(\mathbf{x}, t),
$$

with

$$
\hat{e}_{\boldsymbol{\nabla} \zeta}(\mathbf{x}, t) \equiv \boldsymbol{\nabla} \zeta(\mathbf{x}, t) /|\boldsymbol{\nabla} \zeta(\mathbf{x}, t)| .
$$

From measurements of the scalar field $\zeta(\mathbf{x}, t)$ which are fully resolved in space and time, the scalar field derivative terms $\partial \zeta(\mathbf{x}, t) / \partial t, \boldsymbol{\nabla} \zeta(\mathbf{x}, t)$ and $\nabla^{2} \zeta(\mathbf{x}, t)$ which appear in the scalar transport equation can be computed. This allows $u_{\|}(\mathbf{x}, t)$ to be found by inversion of (1) as

$$
u_{\|}(\mathbf{x}, t)=\left[\frac{1}{\operatorname{ReSc}} \nabla^{2} \zeta(\mathbf{x}, t)-\frac{\partial \zeta(\mathbf{x}, t)}{\partial t}\right]|\nabla \zeta(\mathbf{x}, t)|^{-1},
$$

as first demonstrated by Southerland et al. ${ }^{53}$ This $u_{\|}(\mathbf{x}, t)$ differs between any two points $\mathbf{x}$ and $\mathbf{x}+\delta \mathbf{x}$ due to both the change in the scalar gradient vector direction, and the change in the velocity field $\mathbf{u}(\mathbf{x}, t)$. To first order this can be written

$$
\delta u_{\|}=\mathbf{u}(\mathbf{x}) \cdot \hat{e}_{\nabla \zeta}(\mathbf{x})+(\boldsymbol{\nabla} \mathbf{u}(\mathbf{x}) \cdot \delta \mathbf{x}) \cdot \hat{e}_{\nabla \zeta}(\mathbf{x}+\delta \mathbf{x}),
$$

where the $\delta$ 's represent the differences between the values at $\mathbf{x}+\delta \mathbf{x}$ and $\mathbf{x}$.

The direct inversion scalar imaging velocimetry technique uses this $u_{\|}(\mathbf{x}, t)$ field, together with a smoothness condition, to determine the vector velocity field $\mathbf{u}(\mathbf{x}, t)$ through an iterative process. The starting solution proceeds from an initial assumption that, within small neighborhoods of each point $\mathbf{x}$, the contribution of spatial variations in $\mathbf{u}$ [represented by the second term on the right-hand-side of Eq. (5)] to variations in the measured $u_{\|}$values can be ignored. This is equivalent to assuming that the scalar gradient vector direction $\hat{e}_{\nabla \zeta}$ varies more rapidly than does the velocity vector; this assumption was motivated by the high Schmidt number of currently available fully resolved experimental scalar field measurements. ${ }^{49-51}$ Within such small neighborhoods, the $u_{\|}$'s at three different points are then treated as projections of a single local velocity vector $\mathbf{u}$ onto three different unit vectors $\hat{e}_{\nabla \zeta}$. This leads to the linear system

$$
\left[\begin{array}{c}
\left(u_{\|}\right)_{1} \\
\left(u_{\|}\right)_{2} \\
\left(u_{\|}\right)_{3}
\end{array}\right]=\left[\begin{array}{lll}
\left(\hat{e}_{\nabla \zeta}\right)_{1, x} & \left(\hat{e}_{\nabla \zeta}\right)_{1, y} & \left(\hat{e}_{\nabla \zeta}\right)_{1, z} \\
\left(\hat{e}_{\nabla \zeta}\right)_{2, x} & \left(\hat{e}_{\nabla \zeta}\right)_{2, y} & \left(\hat{e}_{\nabla \zeta}\right)_{2, z} \\
\left(\hat{e}_{\nabla \zeta}\right)_{3, x} & \left(\hat{e}_{\nabla \zeta}\right)_{3, y} & \left(\hat{e}_{\nabla \zeta}\right)_{3, z}
\end{array}\right]\left[\begin{array}{c}
u \\
v \\
w
\end{array}\right],
$$

which is solved for the components $u, v, w$ to yield the starting solution $\mathbf{u}^{(0)}$. Subsequent iterations assume that the velocity $\mathbf{u}$ varies linearly within small neighborhoods of $\mathbf{x}$. Namely, the result $\mathbf{u}^{(k-1)}$ of the $k-1$ iteration is differentiated, and the resulting velocity gradient information is used in the determination of the $k$ th solution $\mathbf{u}^{(k)}$, or

$$
\delta u_{\|}=\mathbf{u}^{(k)}(\mathbf{x}) \cdot \hat{e}_{\nabla \zeta}(\mathbf{x})+\left(\boldsymbol{\nabla} \mathbf{u}^{(k-1)}(\mathbf{x}) \cdot \delta \mathbf{x}\right) \cdot \hat{e}_{\boldsymbol{\nabla} \zeta}(\mathbf{x}+\delta \mathbf{x}) .
$$

The iterations are performed until the $\mathbf{u}^{(k)}(\mathbf{x}, t)$ converge to a self-consistent result. Dahm, Su, and Southerland ${ }^{48}$ have ap- plied this direct inversion technique to test cases with a single $\mathrm{Sc} \gg 1$, passive, conserved scalar field in turbulent flows and have extracted the corresponding velocity and velocity gradient fields.

When applying this technique to experimental scalar field data, the effects of experimental noise and numerical errors in the scalar field derivatives can be dealt with by such measures as writing Eq. (6) for multiple sets of points at each $\mathbf{x}$. However, it is impossible to eliminate entirely the effects of noise on the velocity field results, even in principle. This is because, despite high resolution and signal quality in the underlying scalar field measurements, small but unavoidable errors will be present in the various scalar field derivative terms of the exact conserved scalar transport equation (1). These errors will arise both from experimental noise and from the discretization errors inherent in the numerical differencing operators. The presence of these errors in the scalar field derivatives means that, even if the true velocity field $\mathbf{u}(\mathbf{x}, t)$ were introduced in Eq. (1), the right-hand side of the equation will not in general be exactly zero. As a result, the velocity fields obtained by strict local enforcement of a zero right-hand side in the scalar transport equation, as done in determining $u_{\|}(\mathbf{x}, t)$ by Eq. (4), will be affected by these errors in the scalar field derivatives.

The objective of implementing the scalar imaging velocimetry technique with a formulation that is less sensitive to noise in the scalar field derivative data naturally suggests that the inversion of the scalar transport equation be approached using a minimization technique. The velocity field $\mathbf{u}(\mathbf{x}, t)$ which results from such an approach will not then force a zero right-hand side in Eq. (1), but rather will minimize the integral of the left-hand side of the scalar transport equation over the entire domain, subject to a smoothness constraint as before, and if desired, additional physical constraints.

Since the present scalar imaging velocimetry technique was first presented in 1991 (Dahm, Su and Southerland ${ }^{47,48}$ ), other scalar based velocimetry approaches for fourdimensional measurements in turbulent flows have been proposed. The most closely related is a method of Tokumaru and Dimotakis, ${ }^{46}$ but this does not use the scalar transport equation, and instead finds the velocity field which maximizes a correlation between scalar fields. Similarly, a twoscalar approach has been proposed by Pearlstein and Carpenter, ${ }^{43}$ but this relies on a method for simultaneous measurement of two scalar fields, and requires that the two scalar gradient vectors not align.

The presentation is organized as follows. Section II discusses the formulation of the integral minimization scheme, while Section III presents an assessment of the errors incurred when using the direct inversion and integral minimization techniques to extract velocity fields from essentially noise-free DNS computations. The data used are from the limiting case of unit Schmidt number, and thus pose an especially stringent test on the viability of the integral minimization scheme. Comparison of the results from the integral minimization and direct inversion techniques, as applied to the DNS data, demonstrate that both give velocity fields that reproduce the basic structural features of the underlying DNS 
velocity field. A proper choice of the adjustable weights in the integral minimization technique gives results considerably better than achieved by direct inversion.

\section{INTEGRAL MINIMIZATION SCALAR IMAGING VELOCIMETRY}

\section{A. The integral minimization formulation}

The velocity field sought here is that which minimizes the integral of a functional $E$ over the domain $D$ as

$$
\min _{\mathbf{u}(\mathbf{x}) \in \mathscr{R}^{3} \rightarrow \mathscr{R}^{3}} \int_{D} E\left(u_{1}, u_{2}, u_{3} ; x_{1}, x_{2}, x_{3}\right) d^{3} \mathbf{x},
$$

where the components $u_{i}$ of $\mathbf{u}$ are the dependent variables in $E$, and the components $x_{i}$ of $\mathbf{x}$ are the independent variables. $E$ itself is composed of residuals representing deviations from exact agreement with the scalar transport equation (1), as well as terms which measure the smoothness in the solution $\mathbf{u}(\mathbf{x}, t)$. In general, $E$ in Eq. (8) may be written as the sum of an arbitrary number of such residuals

$$
E \equiv E_{1}+\alpha^{2} E_{2}+\beta^{2} E_{3}+\cdots .
$$

Here each $E_{i} \geqslant 0$ represents a local condition involving the velocity field and possibly the scalar field. The factors $\alpha^{2}$, $\beta^{2}, \ldots>0$ allow control over the relative weights assigned to the individual residuals $E_{i}$ in the minimization functional $E$.

Specification of the terms $E_{i}$ must be made with an eye to the mathematical tools by which Eq. (8) will be solved. The scalar field measurements of Southerland and Dahm ${ }^{49-51}$ will, in the context of scalar imaging velocimetry, yield computational domains as large as $3.3 \times 10^{5}$ points. With three degrees of freedom at each point, corresponding to the three components of the velocity field, the result is a system of approximately one million equations which must be solved at each time $t$. This number of equations can be lowered somewhat in practice through subsampling of the domains, as discussed in Section II C, but the reductions will amount at the most to one order of magnitude. Interest is therefore confined here to those residuals $E_{i}$ which will produce a linear system, so that the simplicity of linear algebraic solution schemes may be applied. A further restriction which is imposed is that the system of equations contain only first and second derivatives of the velocity field, leading to simple and compact discrete difference operators; this restriction allows the matrix associated with the linear system to remain sparse. These conditions, together with the Euler equation of variational calculus [given as Eq. (14)], require that the terms $E_{i}$ be at most second-order in the components of $\mathbf{u}$ and $\nabla \mathbf{u}$.

In this work $E_{1}$ is chosen to be the left-hand side of Eq. (1), and measures the extent to which the scalar field data and any candidate velocity field $\mathbf{u}(\mathbf{x}, t)$ are in "good" agreement with the exact conserved scalar transport equation. Thus, formally

$$
E_{1} \equiv\left(\left[\frac{\partial}{\partial t}+\mathbf{u} \cdot \boldsymbol{\nabla}-\frac{1}{\operatorname{ReSc}} \nabla^{2}\right] \zeta(\mathbf{x}, t)\right)^{2} .
$$

Because only incompressible turbulent flows will be of interest to the present work, a logical second element in our scheme is to penalize deviations from the continuity equation $\boldsymbol{\nabla} \cdot \mathbf{u}=0$. This is represented in strictly non-negative form as

$$
E_{2} \equiv(\boldsymbol{\nabla} \cdot \mathbf{u})^{2} \text {. }
$$

A further condition is needed to specify threedimensional velocity fields uniquely. This ideally would be a direct mathematical representation of a specific physical property of the flow, however the existence of flow properties which can be represented as minimizations of integral quantities is very limited-e.g., to certain simple inviscid, circulation-preserving flows $\left(\right.$ Truesdell ${ }^{54}$ ). Independent of the need to specify velocity fields uniquely in three dimensions, however, is the need to counteract the tendency of $E_{1}$ and $E_{2}$ to introduce artificially small length scales into the velocity field solutions. Such artificial length scales may arise by two mechanisms. The first, which involves $E_{1}$ only, concerns the relative smoothness of the velocity and scalar fields. Velocity fields satisfying only $E_{1}=0$ would in general contain length scales as small as $\lambda_{D}$, the finest length scale of the scalar field. The condition $E_{1}=0$ alone is thus, in general, inconsistent with the true relationship of the finest scales in the velocity and scalar fields. The other mechanism involves both $E_{1}$ and $E_{2}$, and is driven by the effects of experimental noise and discretization errors, which will lead to spurious length scales on the order of the grid spacing of the calculations. These considerations naturally suggest the introduction of some regularizing operator in the inversion process. The use of such regularizing operators is common in inverse problems which involve the analysis of discrete data, and the mathematical basis of the regularization process is well described by Tikhonov and Arsenin. ${ }^{55}$ The residual chosen to supplement $E_{1}$ and $E_{2}$ is one which penalizes nonsmoothness of the velocity field. This is most directly accomplished by minimizing some measure of the magnitude of the velocity gradient components. Among the simplest of these measures is

$$
E_{3} \equiv \nabla \mathbf{u}: \nabla \mathbf{u},
$$

which is simply the sum of squares of the velocity gradient tensor components. Regularizing operators of the form of Eq. (12) are known as "stabilizers" (in particular, $E_{3}$ is a Tikhonov stabilizer of first order), and provide the solutions for $\mathbf{u}$ with the property of mathematical stability in the face of small noise or discretization errors in the initial data.

\section{B. Implications of the smoothness condition}

At this point it is helpful to state the arguments pertaining to the vorticity and scalar diffusion length scales $\lambda_{\nu}$ and $\lambda_{D}$, which represent the finest scales on which spatial gradients can be sustained in the vorticity and scalar fields. As noted by Buch and Dahm, ${ }^{56,57}$ the relationship between these scales provides a measure of the relative smoothness of the velocity and scalar fields and is dependent upon the Schmidt number Sc as

$$
\frac{\lambda_{\nu}}{\lambda_{D}}=\mathrm{Sc}^{1 / 2} \text {. }
$$

The direct inversion implementation of scalar imaging velocimetry was formulated around the assumption that the tar- 
get velocity fields were smoother than the scalar fields. This assumption was driven by the high Schmidt number of the scalar field measurements of Southerland and Dahm ${ }^{49-51}$ (where $\mathrm{Sc} \approx 2075$ ), together with the relation of Eq. (13). The smoothness assumption, rather than being present as an explicit condition satisfied by the velocity field, was present as an implicit consideration in the construction of the direct inversion method. In contrast, the integral minimization implementation allows a smoothness condition to be defined explicitly [here by Eq. (12)], with its weight adjusted to reflect the Schmidt number.

This last point deserves closer inspection. Though the direct inversion technique was formulated with the high Sc, turbulent scalar field data of Southerland and $\mathrm{Dahm}^{49-51}$ in mind, the only Schmidt number regime for which turbulent scalar field data would clearly be insufficient to determine velocity field information is $\mathrm{Sc}<1$; in such cases, the target velocity fields would contain more information than the scalar fields, rendering accurate velocity field determination from scalar field information impossible. For other Schmidt numbers, the scalar fields in principle contain sufficient information to determine the velocity fields, provided that the smoothness condition is defined and weighted appropriately. The "appropriate" weighting for the smoothness condition must take into account both the Schmidt number and the level of experimental and numerical noise present in the scalar field data. The influence of the Schmidt number on the weighting is obvious-larger Sc should require greater values of $\beta^{2}$, i.e., an increased emphasis on the smoothness condition $E_{3}$ relative to the scalar transport condition $E_{1}$. The effect of the noise level, however, is harder to define $a$ priori. Besides affecting the relative weights assigned to $E_{3}$ and $E_{1}$, noise and discretization effects influence the relative weighting of $E_{3}$ and $E_{2}$ as well. Numerical experiments that quantify the effects of various choices for $\alpha^{2}$ and $\beta^{2}$ are given in Section III C.

From inspection of the conditions $E_{2}$ and $E_{3}$, it can be seen that reduction of these residual terms may occur not only by a reduction in the velocity gradients, but also by a general reduction in the velocity vector magnitude throughout the domain. The magnitude of the velocity vector $\mathbf{u}$ therefore "floats." This effect can be countered by a global multiplicative factor which renormalizes the result for $\mathbf{u}$ to guarantee optimal agreement with the scalar transport condition $E_{1}=0$. It may nevertheless be expected that the peak velocity component magnitudes will tend to be underestimated by the integral minimization SIV scheme. However, results from the validation tests of Section III C show that this effect is quite small.

\section{The variational equations}

Once the functional $E\left(u_{1}, u_{2}, u_{3} ; x_{1}, x_{2}, x_{3}\right)$ has been properly specified in terms of the constituent functionals $E_{1}$, $E_{2}$, and $E_{3}$ and the weighting parameters $\alpha^{2}$ and $\beta^{2}$, it remains only to solve Eq. (8) for the velocity field $\mathbf{u}(\mathbf{x}, t)$. This requires first that the system of three equations, corresponding to the three components of the velocity field, be written for each point in the computational domain. From the calculus of variations, the integral of Eq. (8) is stationary if the
Euler characteristic equation for each dependent variable is satisfied. For $E$ as defined here, including only first derivatives of the dependent variables $u_{i}$, the Euler characteristic equation for a given $u_{i}$ has the following general form

$$
\frac{\partial E}{\partial u_{i}}-\sum_{j=1}^{n} \frac{\partial}{\partial x_{j}}\left(\frac{\partial E}{\partial\left(\partial u_{i} / \partial x_{j}\right)}\right)=0
$$

with $n$ the number of independent variables. For Eq. (8), the Euler equation for $u$ is

$$
\frac{\partial E}{\partial u}-\frac{\partial}{\partial x}\left(\frac{\partial E}{\partial u_{x}}\right)-\frac{\partial}{\partial y}\left(\frac{\partial E}{\partial u_{y}}\right)-\frac{\partial}{\partial z}\left(\frac{\partial E}{\partial u_{z}}\right)=0,
$$

with directly analogous results for $v$ and $w$.

With $E$ and its constituent terms $E_{1}, E_{2}$, and $E_{3}$ given in Eqs. (10), (11), and (12), the three Euler equations which result are

$$
\begin{aligned}
& u \zeta_{x}^{2}+v \zeta_{y} \zeta_{x}+w \zeta_{z} \zeta_{x}-\alpha^{2}\left(u_{x x}+v_{y x}+w_{z x}\right)-\beta^{2} \nabla^{2} u \\
& =-\left(\frac{\partial \zeta}{\partial t}-\frac{1}{\operatorname{ReSc}} \nabla^{2} \zeta\right) \zeta_{x} \\
& u \zeta_{x} \zeta_{y}+v \zeta_{y}^{2}+w \zeta_{z} \zeta_{y}-\alpha^{2}\left(u_{x y}+v_{y y}+w_{z y}\right)-\beta^{2} \nabla^{2} v \\
& =-\left(\frac{\partial \zeta}{\partial t}-\frac{1}{\operatorname{ReSc}} \nabla^{2} \zeta\right) \zeta_{y} \\
& u \zeta_{x} \zeta_{z}+v \zeta_{y} \zeta_{z}+w \zeta_{z}^{2}-\alpha^{2}\left(u_{x z}+v_{y z}+w_{z z}\right)-\beta^{2} \nabla^{2} w \\
& =-\left(\frac{\partial \zeta}{\partial t}-\frac{1}{\operatorname{Re~Sc}} \nabla^{2} \zeta\right) \zeta_{z} .
\end{aligned}
$$

The determination of the velocity field $\mathbf{u}(\mathbf{x}, t)$ then involves writing Eqs. (16), (17), and (18) for each of the discrete points in the domain, with the velocity derivative terms represented by discrete operators. The equations are then concatenated to form a linear system in the velocity components, and the resulting system is solved for $\mathbf{u}(\mathbf{x}, t)$.

The scalar field derivatives are evaluated from the scalar field data using linear central differences and, in the case of second derivatives, the operators resulting from successive applications of central differencing. The velocity derivative terms in Eqs. (16), (17), and (18) are discretized using the same difference operators. At the limits of the domain, reflective boundary conditions are used [e.g., at $x=0$, $\left.u_{i}(\Delta x)=u_{i}(-\Delta x)\right]$. The resulting vanishing derivative across the boundary is consistent with the minimization of velocity gradients through Eq. (12).

As a final note, the linear system $\mathbf{A u}=\mathbf{b}$ which results from Eqs. (16), (17), and (18) is solved using an iterative method (Eisenstat $e t a l{ }^{58}$ ) similar to the method of conjugate gradients. The solution convergence is conditioned upon the residual $|\mathbf{A u}-\mathbf{b}|$. For the results presented here, the initial solution used is simply $\mathbf{u} \equiv 0$. For velocity determination on a time series of scalar field measurement data, such as will be seen in Part II ${ }^{52}$ increased speed of convergence is pursued by using the velocity field result $\mathbf{u}(\mathbf{x}, t)$ for a given time step as the initial estimate for the velocity field at the subsequent step. 


\section{Nature of the extremum}

We show here that the variational Euler equations (16), (17), and (18) isolate a local extremum, and that this extremum is a minimum. This will rely on the second variation of the integral. Because the general theory of the second variation for functions involving three dependent variables and three independent variables is not well developed, this also provides an opportunity to illustrate some of the key mathematical principles used in the integral minimization form of scalar imaging velocimetry. Fox $^{59}$

The integral in question here is (the notation follows

$$
I=\int_{D} E(u, v, w, \boldsymbol{\nabla} u, \boldsymbol{\nabla} v, \boldsymbol{\nabla} w ; x, y, z) d x d y d z
$$

where the explicit dependence on the three components of $\mathbf{u}$ and the nine components of $\mathbf{\nabla u}$ is shown. The (weak) variations of interest will have the form

$$
\mathbf{u}=\mathbf{s}(\mathbf{x})+\epsilon \mathbf{t}(\mathbf{x})
$$

whose components are labeled

$$
u_{i}=s_{i}(\mathbf{x})+\epsilon t_{i}(\mathbf{x}) .
$$

Under the influence of the variations described by Eq. (20), the integral $I$ will assume the form

$$
I=I_{s}+\delta I_{s}
$$

where $I_{s}$ is defined by

$$
I_{s}=\int_{D} E\left(s_{1}, s_{2}, s_{3}, \nabla s_{1}, \nabla s_{2}, \nabla s_{3} ; x, y, z\right) d x d y d z
$$

and thus $I=I_{s}+\delta I_{s}$ has the form

$I_{s}+\delta I_{s}=\int_{D} E\left(s_{1}+\epsilon t_{1}, \ldots, \nabla\left(s_{1}+\epsilon t_{1}\right), \ldots ; x, y, z\right) d x d y d z$

where $\delta I_{s}$ is itself defined as

$$
\delta I_{s}=\epsilon I_{1}+\frac{\epsilon^{2}}{2} I_{2}+O\left(\epsilon^{3}\right) .
$$

Here $I_{1}$ is referred to as the first variation and $I_{2}$ as the second variation.

In order that $I_{s}$ represents a minimum for the integral $I, \delta I_{s}$ must be positive for all sufficiently small values of $\epsilon$. It is therefore sufficient that $I_{1}=0$ and $I_{2}>0$ in Eq. (25). That the first condition, $I_{1}=0$, is observed by the functional $E$ and the velocity field result $\mathbf{u}$ is guaranteed by the Euler characteristic equations (16), (17), and (18). These equations were specified through application of the general result for the Euler equations (14). The theory of the first variation by which Eq. (14) may be derived is very well described in many texts. In contrast, the general theory for the second variation which would be relevant to this form of $I$ (involving three dependent and three independent variables) is analytically very involved. Instead, the form of the second variation for this particular problem is derived below.

$I_{2}$ for this problem is defined by

$$
\begin{aligned}
I_{2}= & \int_{D}\left[t_{i} t_{j} \frac{\partial^{2} E}{\partial u_{i} \partial u_{j}}+\frac{\partial t_{i}}{\partial x_{k}} \frac{\partial t_{j}}{\partial x_{l}} \frac{\partial^{2} E}{\partial\left(\partial u_{i} / \partial x_{k}\right) \partial\left(\partial u_{j} / \partial x_{l}\right)}\right] \\
& \times d x d y d z .
\end{aligned}
$$

This equation reflects the dependence of $E$ on the velocity components $u_{i}$ and their first derivatives $\partial u_{i} / \partial x_{i}$, and also reflects the absence of any cross terms involving both the velocity components and their derivatives. Applying this to the specific form of $E$ given by Eqs. (9), (10), (11), and (12), this becomes

$$
I_{2}=\int_{D}\left[(\mathbf{t} \cdot \boldsymbol{\nabla} \zeta)^{2}+\alpha^{2}(\boldsymbol{\nabla} \cdot \mathbf{t})^{2}+\beta^{2} \boldsymbol{\nabla} \mathbf{t}: \boldsymbol{\nabla} \mathbf{t}\right] d x d y d z .
$$

Having applied the Euler characteristic equations to the first variation, the integral of $E$ over the domain $D$ is minimized if this $I_{2}$ is positive, independent of the choice of $\mathbf{t}(\mathbf{x})$ in Eq. (20). This is manifestly satisfied except for the uninteresting case of $\mathbf{t} \equiv 0$. The simplicity of the form of Eq. (27), and the similarity of the integrand to the functional $E$ itself, can be traced directly to the original formulation of $E$ in terms of quantities of at most second order in the components of $\mathbf{u}$ and $\boldsymbol{\nabla u}$. That restriction was made to guarantee simplicity in the solution method, and returns here to simplify the analysis as well.

\section{A DNS-BASED TEST OF SCALAR IMAGING VELOCIMETRY}

This section presents results from the application of this integral minimization scalar imaging velocimetry technique to a direct numerical simulation (DNS) of $\mathrm{Sc}=1$ passive scalar mixing in a turbulent flow. The velocity fields obtained by the scalar imaging velocimetry technique can be directly compared with the actual velocity fields used in the simulation, allowing for rigorous quantification of the accuracy of the technique. Section III A discusses properties of the DNS data, and relates these properties to the experimental scalar field measurements of Southerland and Dahm. ${ }^{49-51}$ In Section III B the choice of $\alpha^{2}$ and $\beta^{2}$ for these $\mathrm{Sc}=1$ data is discussed. Results from the application of the integral minimization formulation of SIV to these DNS data are presented in Section III C. For comparison, that section also presents results from the application of the direct inversion technique $^{48}$ to the DNS data.

\section{A. Properties of the DNS data}

The DNS scalar and velocity field data used here are from a simulation of the turbulent mixing of a dynamically passive $\mathrm{Sc}=1$ conserved scalar quantity by Mell et al. ${ }^{60-62}$ The turbulent flow was decaying, homogeneous, isotropic and incompressible, and its evolution was computed in a $128^{3}$ volume. At the time chosen for this test, the Taylor scale Reynolds number $\operatorname{Re}_{\lambda}$ has decayed to 23 from its initial value of 36. A sample scalar field plane from this simulation is shown in Fig. 1.

Because the aim of this study is to assess the applicability of the integral minimization scalar imaging velocimetry technique to actual laboratory turbulent flows, it is important that the scalar field information used be representative of the 

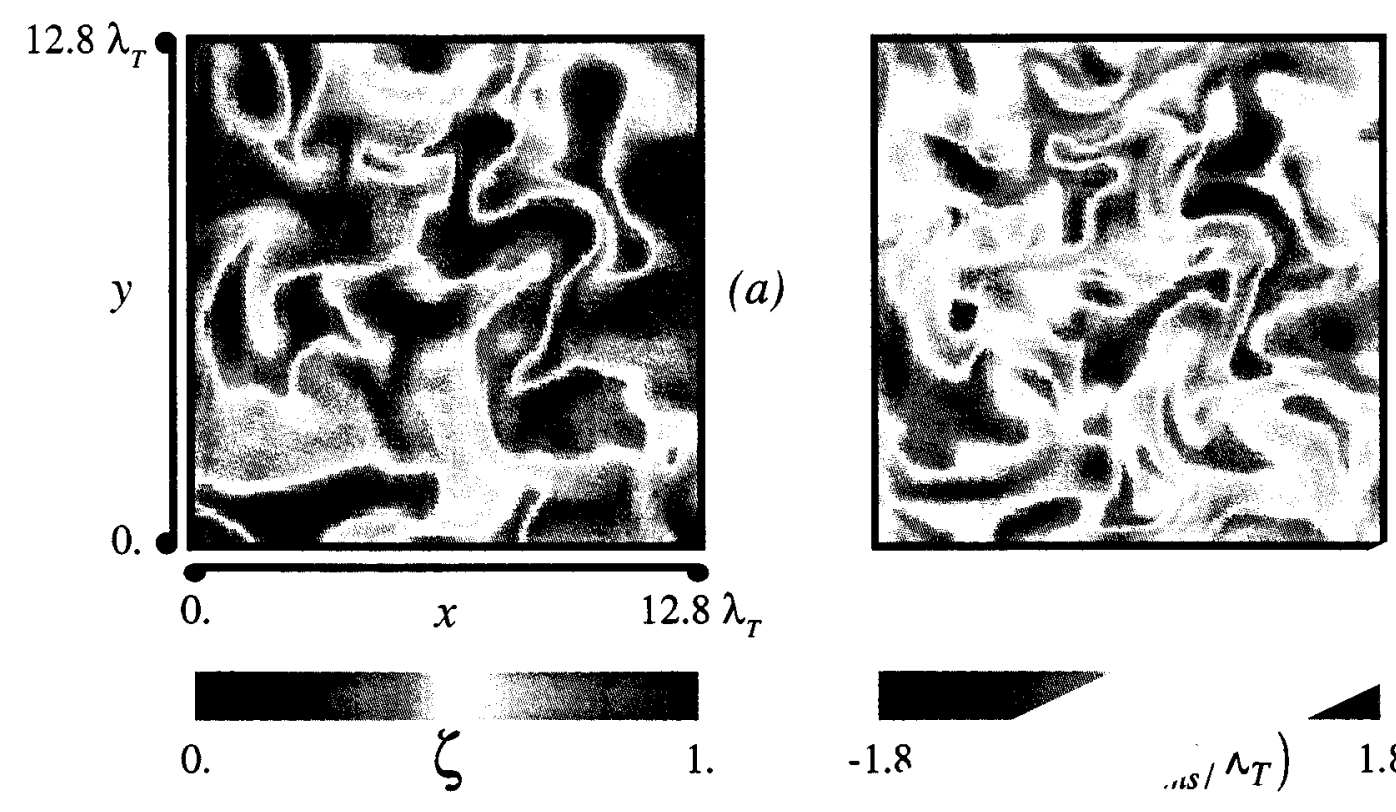

(b)
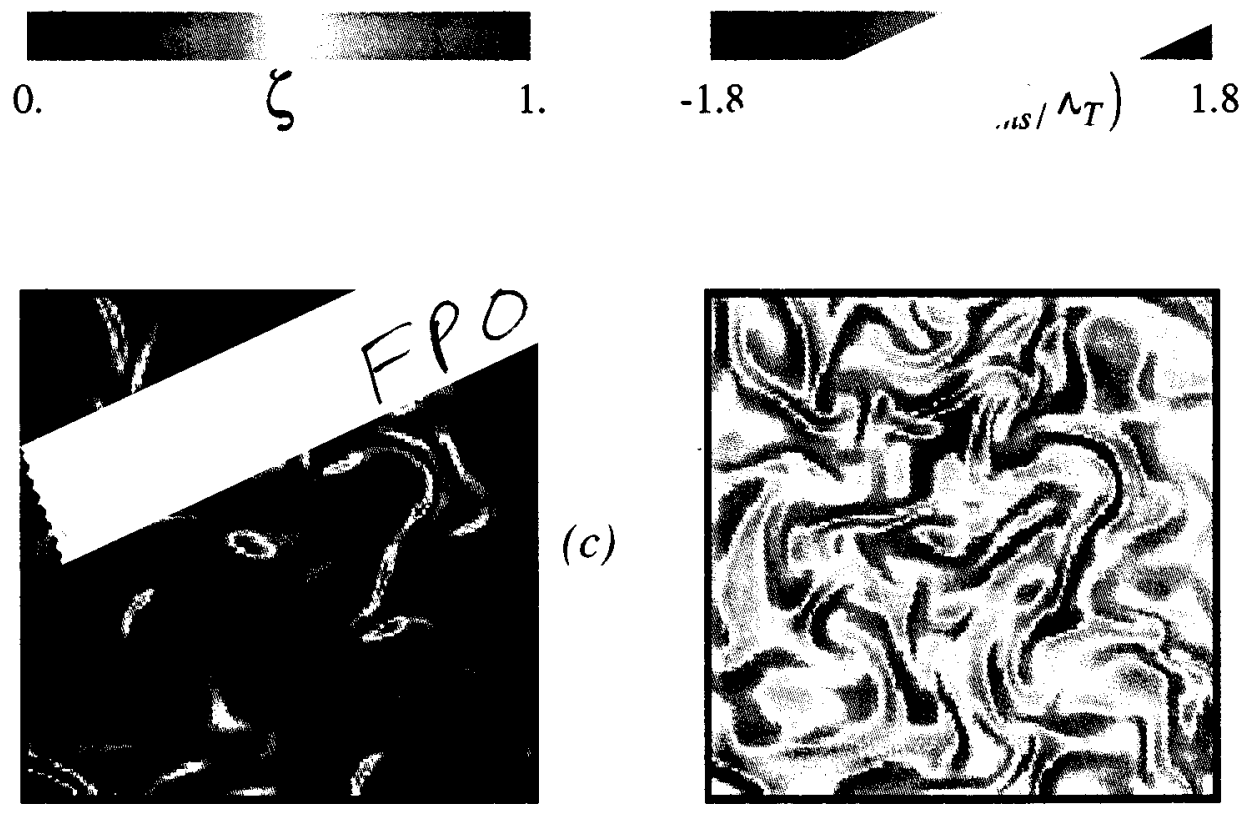

(c)
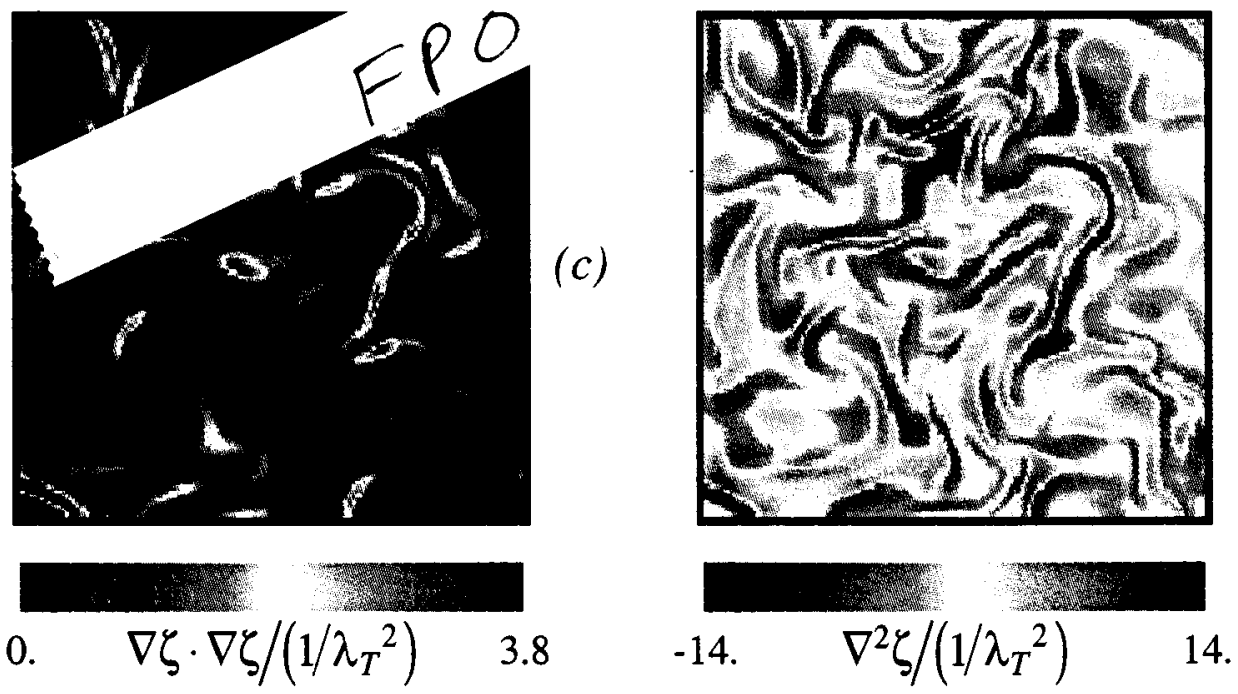

FIG. 1. Scalar field and scalar field derivative information from the simulations of Mell et al. (Refs. 60, 61, and 62). (a) The scalar field $\zeta(\mathbf{x}, t)$. (b) The time derivative field $\partial \zeta(\mathbf{x}, t) / \partial t$. (c) The scalar energy dissipation rate field $\nabla \zeta \cdot \nabla \zeta(\mathbf{x}, t)$. (d) The Laplacian field $\nabla^{2} \zeta(\mathbf{x}, t)$.

available scalar field measurements. For this reason, the original $128^{3}$ data volumes are subsampled spatially to 128 $\times 128 \times 15$, to approximate the relative dimensions of the experimental scalar field data volumes as well as to match the levels of spatial resolution. The DNS data are also subsampled in time, as explained below, to make the temporal resolution comparable to that of the experimental data.

For this simulation of homogeneous, isotropic flow, the reference length and velocity scales $l^{*}$ and $u^{*}$ will be the Taylor length scale $\lambda_{T}$ and the rms velocity $\langle\mathbf{u} \cdot \mathbf{u}\rangle^{1 / 2}$, respectively. The scaled grid spacing in the simulation is $\Delta x=\Delta y=\Delta z \approx 0.1 \lambda_{T}$. The time step between data volumes is markedly smaller, namely $\Delta t \approx 0.0035 \lambda_{T} /|\mathbf{u}|_{\mathrm{rms}}$. The reason for the much higher time resolution is that the simulation used a fixed time step, which was chosen to resolve ad- equately the time scales of the initial turbulence and overresolves the flow as the turbulence decays with time. To match the spatial and temporal resolutions to a greater degree, only every twentieth simulation time step was used in forming the central differences from which the time derivative was calculated. The resulting time separation was $\Delta t \approx 0.070 \lambda_{T} /|\mathbf{u}|_{\mathrm{rms}}$, which, when compared with the grid spacing $\Delta x$, mimics the roughly equivalent levels of spatial and temporal resolution characteristic of the experimental scalar field data of Southerland and Dahm. ${ }^{49-51}$

\section{B. Numerical parameters}

The variational Euler equations (16), (17), and (18) involve the factors $\alpha^{2}$ and $\beta^{2}$, which allow the relative weights 
of the three residuals $E_{1}, E_{2}$, and $E_{3}$ [Eqs. (10), (11), and (12)] to be adjusted according to the characteristics of the given scalar field data. The value of $\beta^{2}$, which describes the importance of the smoothness residual $E_{3}$ relative to the scalar transport residual $E_{1}$, is chosen to reflect the relation between the smoothness of the velocity and scalar fields. As mentioned in Sections II A and II B, satisfaction of the scalar transport condition alone can be expected to admit velocity fields with length scales as fine as the smallest length scales in the scalar field. Since for these $\mathrm{Sc}=1 \mathrm{DNS}$ data the finest scales of the velocity and scalar fields are nominally equivalent, we anticipate that ideally $\beta^{2} \ll 1$. The continuity residual $E_{2}$ may itself permit artificially small length scales, on the order of the grid spacing of the calculations, to enter the velocity field results. The need to counteract this effect suggests that $\alpha^{2}$ and $\beta^{2}$ will be of the same order.

However, a priori specification of $\alpha^{2}$ and $\beta^{2}$ beyond these guidelines is not possible. In fact, even if the optimal values for $\alpha^{2}$ and $\beta^{2}$ were known for a given Schmidt number, it is not apparent how these might be properly scaled to a different Sc. The only guarantee is that increasing (decreasing) values of Sc will be accompanied by increases (decreases) in the optimal value of $\alpha^{2}$ and $\beta^{2}$. The result is that the optimal values of these parameters for a given Schmidt number must be determined by numerical experiments. The criterion used for determining whether the optimal $\beta^{2}$ has been found is to require that the finest length scale of the velocity field results be consistent with the estimate for $\lambda_{\nu}$. The optimal $\alpha^{2}$ is then chosen as the highest value which can be used without spurious, small scale discretization errors becoming evident. This procedure is simplified by the relative insensitivity of the integral minimization formulation to changes in $\alpha^{2}$ and $\beta^{2}$; for example, decreasing values of the smoothness parameter $\beta^{2}$ increase the amount of fine scale information in the results without significant effect on the larger structures. The effect of small changes (i.e., within 1 order of magnitude) in $\alpha^{2}$ and $\beta^{2}$ on the velocity field results is indeed virtually negligible. This is seen in Fig. 2, which shows the effect of changes in $\beta^{2}$ (with the ratio $\beta / \alpha$ fixed at $2 / 3$ ) on the results for $\mathbf{u}$ and $\boldsymbol{\nabla} \mathbf{u}$ in terms of their correlations with the known DNS fields. [The correlation measure used will be defined by Eq. (28).] Also shown in the figure is the effect of changes in $\alpha^{2}$ upon the same correlations, with a fixed value of $\beta^{2}=2 \cdot 10^{-4}$. Based on these numerical experiments, the values of $\alpha^{2}$ and $\beta^{2}$ chosen for the results of Section III C are, respectively, $5 \cdot 10^{-4}$ and $2 \cdot 10^{-4}$. From Eq. (9), these choices of $\alpha^{2}$ and $\beta^{2}$ mean that the velocity field is primarily constrained to satisfy the scalar transport residual $E_{1}$, with only a small weight assigned to either the continuity or smoothness residuals $E_{2}$ and $E_{3}$. The very small weight on the smoothness residual is a consequence of the inherent smoothness of the $(\mathrm{Sc}=1)$ scalar field data.

\section{Results}

The scalar field derivatives $\partial \zeta / \partial t, \nabla \zeta$, and $\nabla^{2} \zeta$ provide the inputs to the scalar imaging velocimetry technique. These are found through linear central differences from the scalar field data, and are shown in Fig. 1 along with the corresponding scalar field plane. The velocity component fields $u, v$

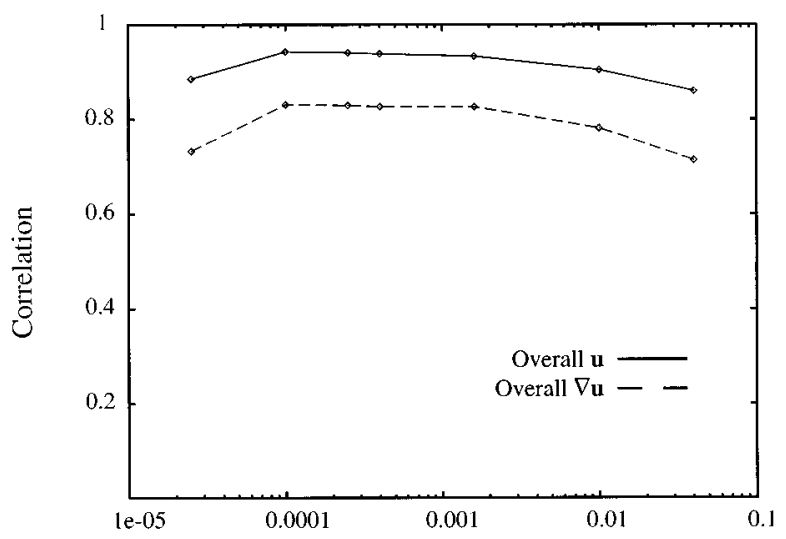

(a)

$\beta^{2}$

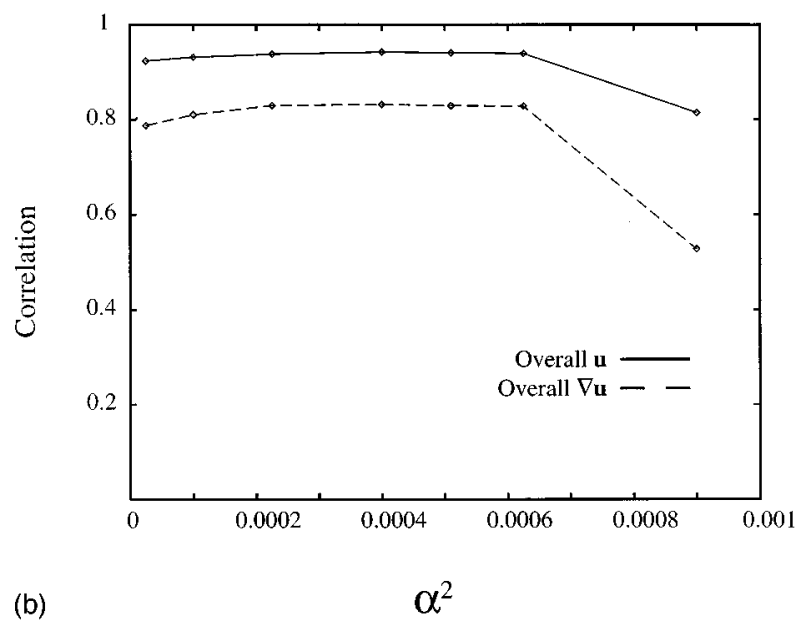

FIG. 2. Sensitivity of the integral minimization SIV results to changes in the weighting parameters $\alpha^{2}$ and $\beta^{2}$. Shown are the velocity component SIVDNS correlations (incorporating the three components of $\mathbf{u}$ ) and velocity gradient component correlations (incorporating the nine components of $\mathbf{\nabla u}$ ) corresponding to the planes displayed in the preceding figures, for different values of $\beta^{2}$ (a), with a fixed ratio of $\beta / \alpha=2 / 3$ imposed. Also shown are (b) the SIV-DNS correlations for different values of $\alpha^{2}$, with $\beta^{2}=2 \cdot 10^{-4}$. The results presented in this paper were found with values of $\alpha^{2}=5 \cdot 10^{-4}$ and $\beta^{2}=2 \cdot 10^{-4}$.

and $w$ which result from these derivative fields for both the integral minimization and direct inversion SIV approaches are shown in Figs. 3, 4, and 5, together with the exact DNS fields for comparison. The normalization of all quantities shown is by the same scale factor, namely the rms velocity magnitude $|\mathbf{u}|_{\mathrm{rms}} \equiv\langle\mathbf{u} \cdot \mathbf{u}\rangle^{1 / 2}$ from the DNS fields. This allows direct comparisons of the integral minimization and direct inversion results with each other as well as with the DNS fields. It is evident, even from a visual inspection of these figures, that both SIV formulations reproduce the basic structural features of the DNS fields. It is also apparent that the integral minimization approach yields results that are considerably more accurate than those obtained by the direct inversion approach. Since the DNS scalar field data are essentially free of noise, the improvements obtained with the integral minimization formulation appear to be traceable to two primary considerations. One of these is the ability to include the continuity constraint $E_{2}$ in the procedure for determining the 


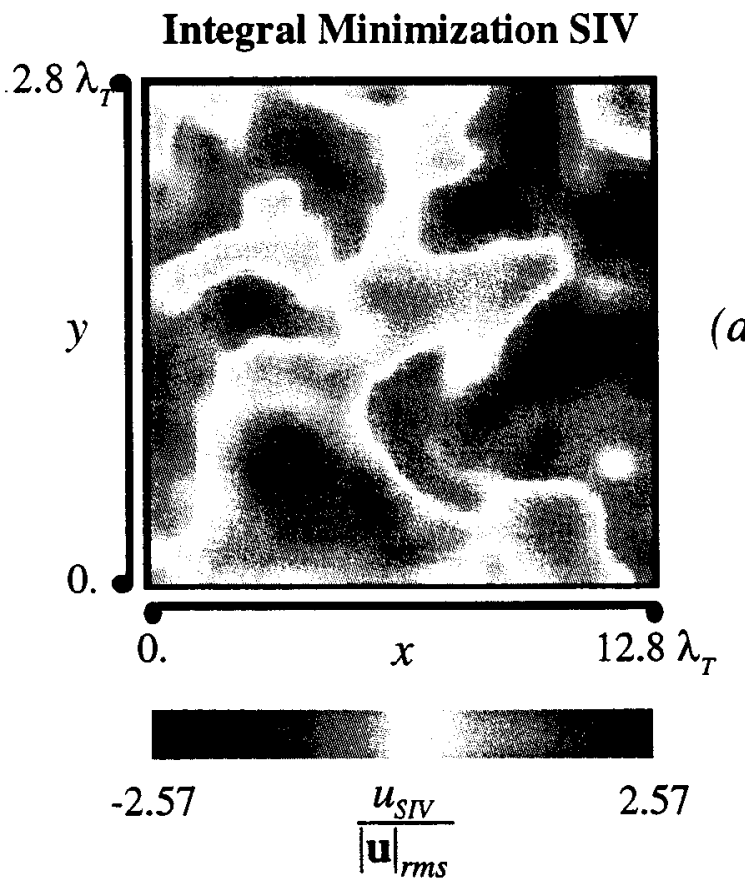

\section{Direct Inversion SIV}

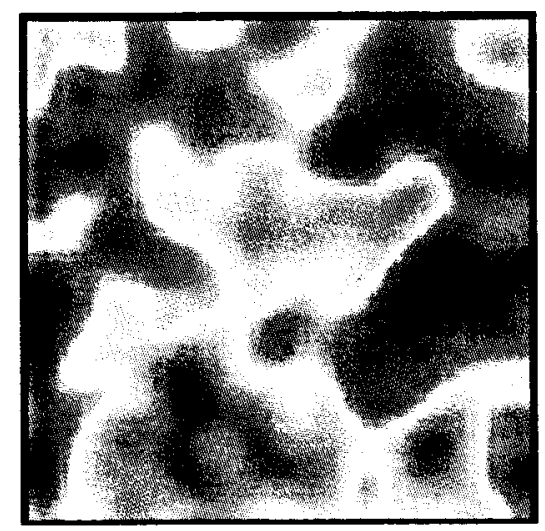

(b)

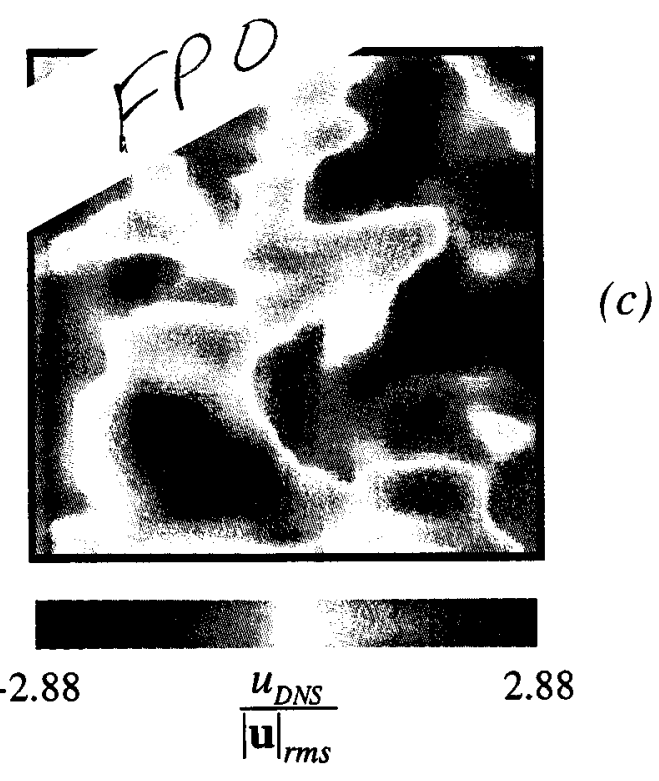

FIG. 3. Comparison of the SIV results for $\mathbf{u}(\mathbf{x}, t)$ with the DNS fields. (a) The integral minimization SIV result for the $u$-component. (b) The direct inversion SIV result. (c) The DNS field. Velocity component correlations are presented in Table I.

velocity fields. The other is the more direct fashion in which the smoothness constraint enters the integral minimization formulation.

The improvements seen in the integral minimization results over those obtained by the direct inversion formulation are quantified in Table I. This table gives the correlation between the DNS fields and those obtained from both the integral minimization and direct inversion approaches. The values shown are standard moment correlations $R$, defined for any two fields $u_{i}$ and $u_{j}$ as

$$
R_{u_{i} u_{j}} \equiv \frac{\left\langle u_{i}^{\prime} u_{j}^{\prime}\right\rangle}{\left(u_{i}^{\prime}\right)_{\mathrm{rms}}\left(u_{j}^{\prime}\right)_{\mathrm{rms}}} .
$$

The correlations between the SIV and DNS velocity component fields $u, v$, and $w$ are typically 0.96 with the integral minimization approach, but only 0.84 with the direct inversion approach. Similarly, when the velocity gradient component fields from the two approaches are compared with the corresponding DNS fields, the resulting overall correlation is 0.84 with the integral minimization approach, but only 0.53 with the direct inversion approach. As a further test on the accuracy of these results, Table I also includes measures of the agreement of the velocity field solutions with the continuity condition $\boldsymbol{\nabla} \cdot \mathbf{u}=0$ of incompressible flow. The quantity labeled " $x$-divergence" is the correlation between $(\partial u / \partial x)$ and $(-\partial v / \partial y-\partial w / \partial z)$, which assumes a value of 1 


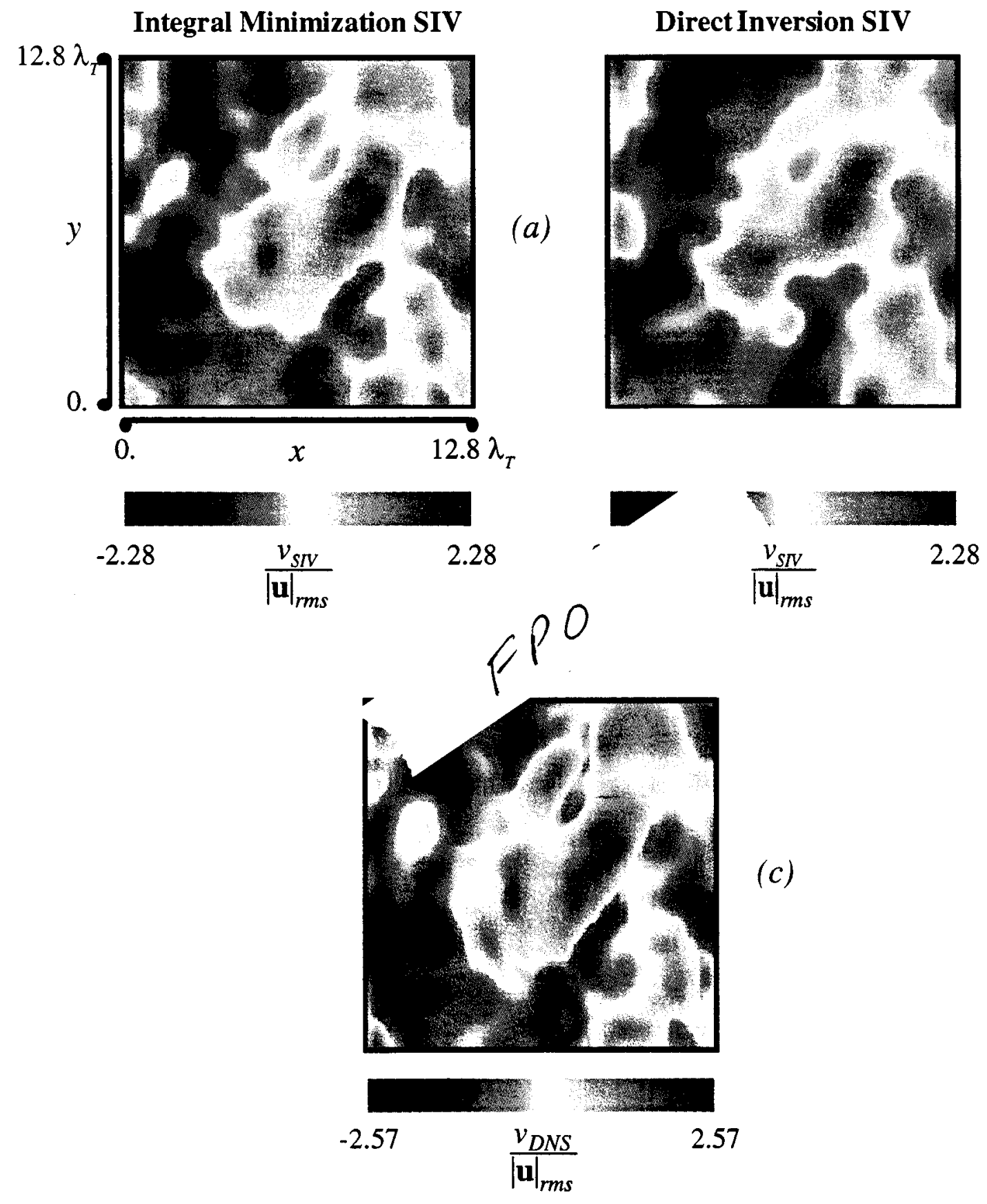

FIG. 4. The integral minimization SIV result for the $v$-component (a), the direct inversion result (b), and (c) the DNS field. Correlations are in Table I.

when continuity is exactly satisfied. The $y$ - and $z$-divergence quantities are similarly defined. These correlations lie in the range $0.85-0.89$ for the integral minimization results, with much lower values $(0.50-0.60)$ for the direct inversion results. These correlations clearly demonstrate that the integral minimization approach is better able to determine the structure of these velocity fields from the original $\mathrm{Sc}=1$ turbulent scalar field data.

While correlations of the type in Table I allow assessment of the structural similarity (i.e., phase agreement) between the DNS and SIV fields, they are insensitive to differences in the absolute magnitudes of these fields. For this reason, Fig. 6 presents distributions of the values of the velocity components $u, v$, and $w$ for the integral minimization
SIV and DNS fields. These distributions are all normalized by the same scale factor $|\mathbf{u}|_{\mathrm{rms}}$. In each case, the SIV and DNS distributions show strikingly similar form. These distributions also show a result anticipated in Section II B, namely that the peak velocity component values for the integral minimization results slightly underestimate the true peak values. These minor discrepancies in peak values have been taken into account in the choice of color assignments in Figs. $3-5$.

While the DNS data as used in this validation study emulate the resolution characteristics of currently available experimental scalar field measurements, they are free of the random noise which is present in such experimental measurements of $\zeta(\mathbf{x}, t)$. Figure 7 shows the effect on the SIV 
Integral Minimization SIV

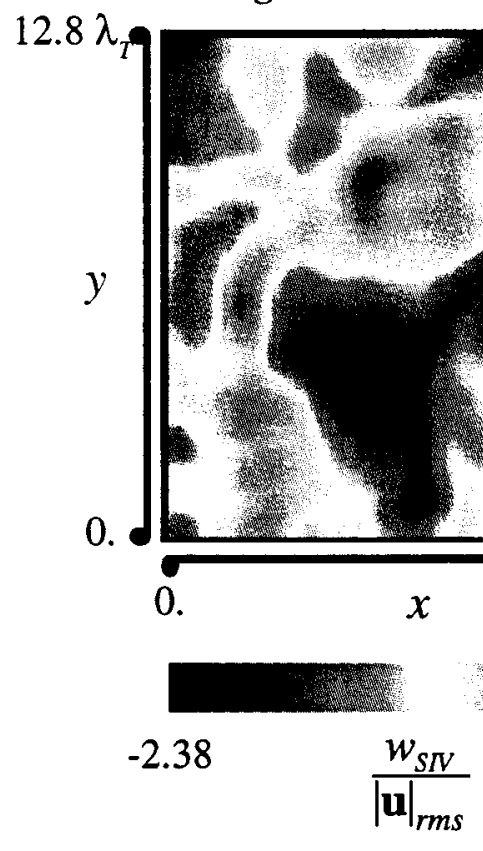

Direct Inversion SIV

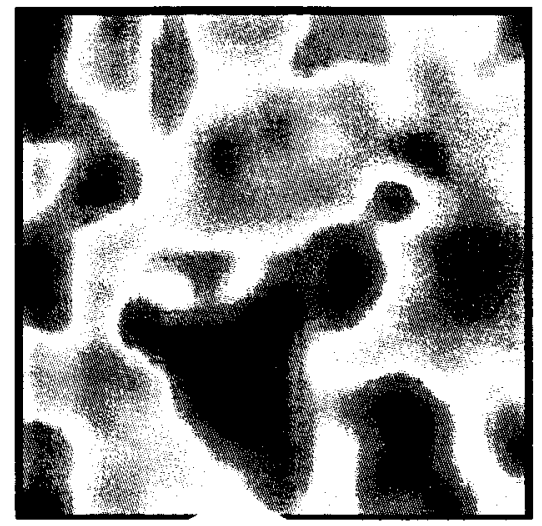

(b) (a)

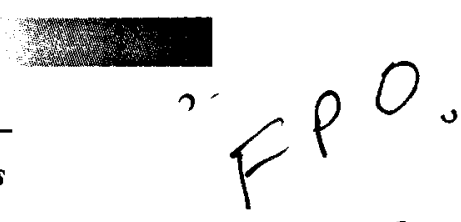

DNS

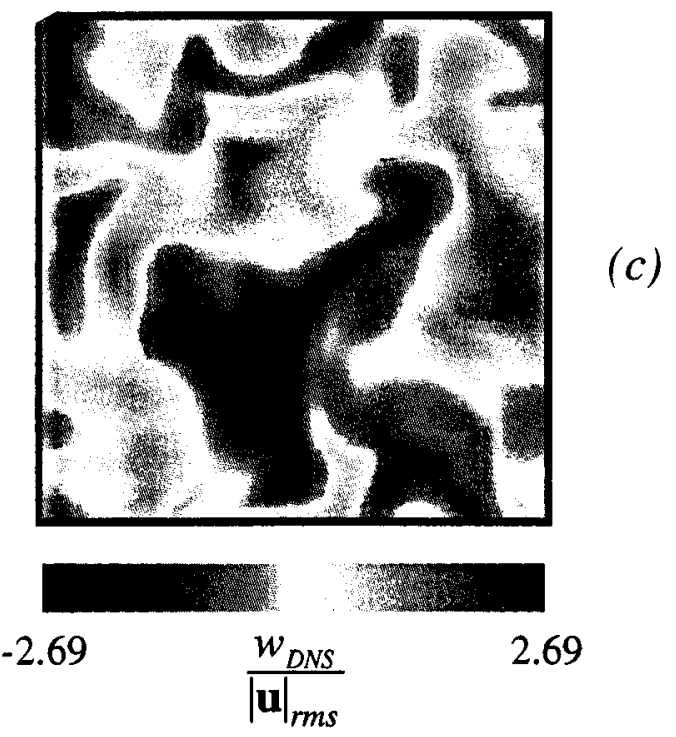

FIG. 5. The integral minimization SIV result for the $w$-component (a), the direct inversion result (b), and (c) the DNS field. Correlations are in Table I.

results for $\mathbf{u}$ and $\boldsymbol{\nabla} \mathbf{u}$ of random (Gaussian) noise added to the DNS scalar fields. The signal-to-noise level indicated is the ratio of the maximum signal level in the DNS scalar fields to the rms value of the Gaussian noise distribution added to those fields. Shown in the figure are the correlations of $\mathbf{u}$ and $\nabla \mathbf{u}$ between the DNS fields and SIV results, using the same correlation measure as Fig. 2. The values of the weighting factors $\alpha^{2}$ and $\beta^{2}$ used are, as before, $5 \cdot 10^{-4}$ and $2 \cdot 10^{-4}$, respectively.

Figure 7 demonstrates that the $\mathbf{u}$ and $\boldsymbol{\nabla u}$ correlations are largely insensitive to scalar field noise throughout a range of noise levels, from a low noise level with an associated signal-to-noise level of 600 , up to a signal-to-noise level of
10 (corresponding to the unrealistic case of effectively 3-bit data). The results in Fig. 7 should be viewed relative to the noise estimates in current practical four-dimensional scalar field measurements. ${ }^{50,51}$ At lower signal levels (the "camera noise" regime), the noise level in those measurements is roughly constant at 0.8 digital levels out of a maximum 256, so the signal-to-noise level is a linear function of the signal. At higher signal levels (the "shot noise" regime) the signalto-noise level goes as the square root of the signal, with a maximum noise level of roughly 1.3 digital levels. At their maximum signal level, Southerland and Dahm estimate a signal-to-noise value of slightly over 200. This level is indicated in Fig. 7. In fact, because the Gaussian noise distribu- 
TABLE I. Correlations between the SIV results, for both the direct inversion and integral minimization formulations, and the actual DNS fields. The correlation measure used in given by Eq. (28). The overall correlation shown encompasses all nine components of the tensor $\nabla \mathbf{u}$.

\begin{tabular}{lcc}
\hline \hline & $\begin{array}{c}\text { Direct } \\
\text { inversion }\end{array}$ & $\begin{array}{c}\text { Integral } \\
\text { minimization }\end{array}$ \\
\hline$u$-component & 0.869 & 0.964 \\
$v$-component & 0.827 & 0.961 \\
$w$-component & 0.826 & 0.946 \\
$\partial u / \partial x$ & 0.604 & 0.860 \\
$\partial u / \partial y$ & 0.604 & 0.847 \\
$\partial u / \partial z$ & 0.550 & 0.806 \\
$\partial v / \partial x$ & 0.538 & 0.849 \\
$\partial v / \partial y$ & 0.492 & 0.832 \\
$\partial v / \partial z$ & 0.413 & 0.867 \\
$\partial w / \partial x$ & 0.599 & 0.849 \\
$\partial w / \partial y$ & 0.535 & 0.816 \\
$\partial w / \partial z$ & 0.436 & 0.784 \\
Overall $\partial u_{i} / \partial x_{j}$ & 0.538 & 0.835 \\
$x$-divergence & 0.557 & 0.887 \\
$y$-divergence & 0.502 & 0.865 \\
$z$-divergence & 0.598 & 0.855 \\
\hline \hline
\end{tabular}

tion used is independent of the scalar value, the noise added overestimates the experimental noise at low signal levels. Thus the typical experimental noise level indicated in Fig. 7 represents a worst-case assessment of the influence of noise. Despite this, it can be seen that at that noise level the overall SIV-DNS u correlation falls minimally to 0.92 from its noise-free value of 0.94

\section{CONCLUSIONS}

The high values of these SIV-DNS correlations (typically 0.96 for the velocity components and 0.84 for the velocity gradient components), and their insensitivity to anticipated levels of realistic experimental noise, offer strong evidence that the integral minimization scalar imaging velocimetry technique can accurately determine threedimensional velocity fields from turbulent scalar field data. The errors in satisfying continuity, shown in Table I, are significantly smaller for the minimization technique, which explicitly penalizes departures from the mass conservation condition. These errors should be viewed in the context of current hot-wire techniques used in the measurement of turbulent velocity gradients. In those studies, the continuity correlation values do not exceed 0.7 (cf. Tsinober, Kit, and $\operatorname{Dracos}^{22}$ ), in contrast to the values in excess of 0.85 demonstrated by the integral minimization technique.

It is significant that these results were found from scalar field data at unit Schmidt number, for which the scalar and velocity fields have nominally equivalent levels of detail. Thus the scalar field contains no redundancy of information which would assist in determining the velocity fields. In contrast, for high Schmidt numbers, the disparity in the characteristic length scales of the velocity and scalar fields dictates that the scalar field data carries more information than the target velocity field. For example, for the $\mathrm{Sc} \approx 2075$ scalar field data in Part II, the finest velocity gradient length scale
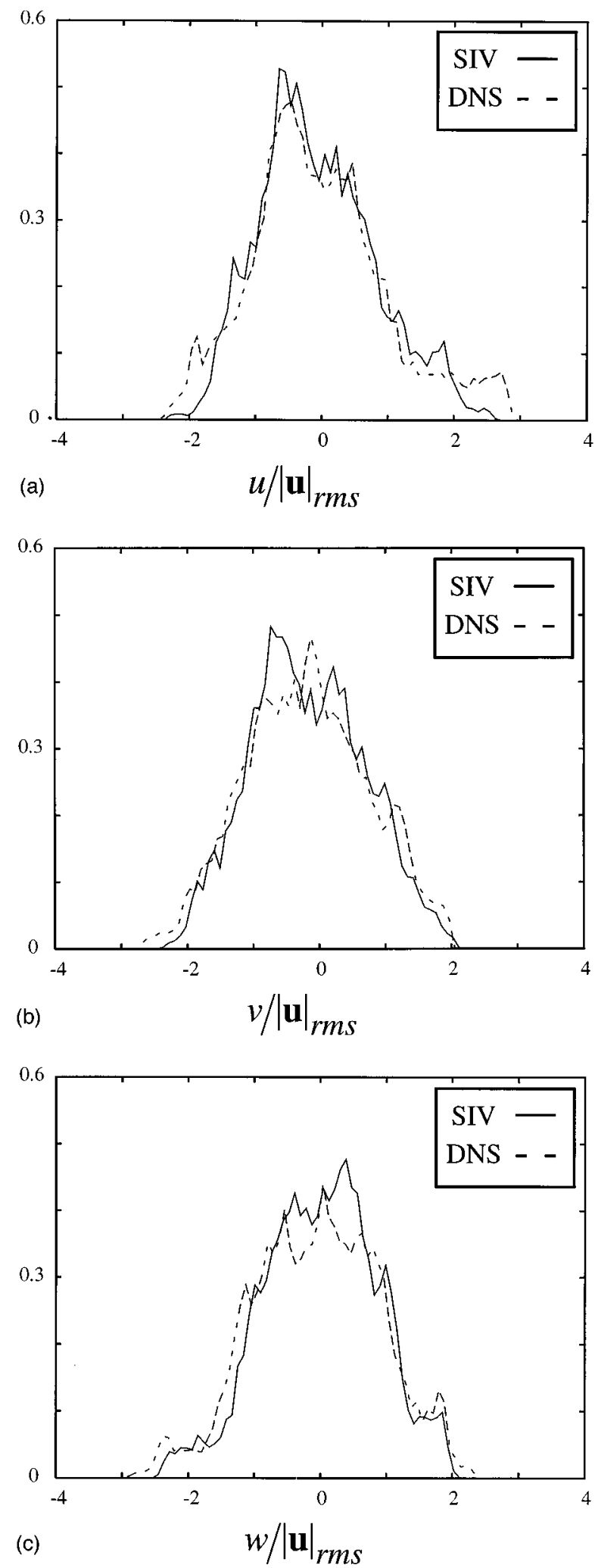

FIG. 6. Distributions of the velocity component values, for both the integral minimization SIV results and the DNS fields. The SIV results show good agreement with the DNS fields, save for an underestimation of the highest velocity magnitudes. (a) The $u$-component, (b) the $v$-component, (c) the $w$-component.

$\lambda_{\nu}$ is roughly 45 times larger than the scalar gradient length scale $\lambda_{D}$. This suggests that this integral minimization SIV technique will yield results of at least the level of accuracy achieved for this $\mathrm{Sc}=1$ DNS validation test, when ap- 


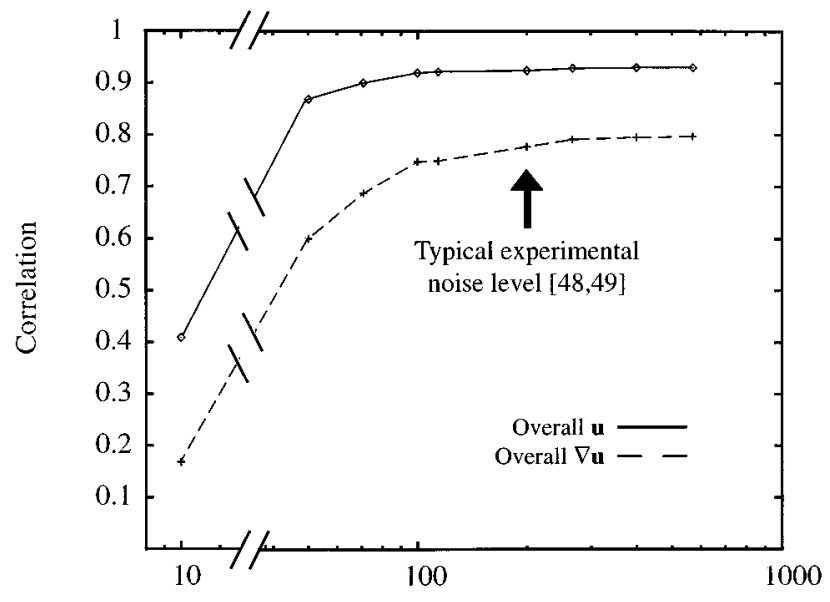

Signal-to-Noise Level

FIG. 7. The effect of artificially introduced scalar field noise on the integral minimization SIV results. Shown are the SIV-DNS correlations for $\mathbf{u}$ and $\boldsymbol{\nabla u}$ for different values of the signal-to-noise level. The signal-to-noise level is defined here in terms of the maximum scalar field signal and the rms of the Gaussian random noise distribution.

plied to the experimental high Sc scalar field data as is done in Part II. ${ }^{52}$

\section{ACKNOWLEDGMENTS}

The first author was supported by a François-Xavier Bagnoud Fellowship from the Association FXB. The DNS data were generously provided by Dr. W. E. Mell and Profs. G. Kosàly and J. J. Riley of The University of Washington. Early development of the scalar imaging velocimetry technique was supported by the Air Force Office of Scientific Research (AFOSR) under Grant No. F49620-92-J-0025.

${ }^{1}$ L. S. G. Kovasznay, "Turbulence measurements," in Physical Measurements in Gas Dynamics and Combustion, Vol. 10 (Princeton University, Princeton, 1954).

${ }^{2}$ S. Corrsin and A. L. Kistler, "The free-stream boundaries of turbulent flows," NACA TN 3133 (1954).

${ }^{3}$ W. W. Willmarth and S. S. Lu, "Structure of the Reynolds stress near the wall," J. Fluid Mech. 55, 65 (1972).

${ }^{4} \mathrm{~W}$. W. Willmarth and T. J. Bogar, "Survey and new measurements of turbulent structure near the wall," Phys. Fluids 20, S9 (1977).

${ }^{5}$ E. G. Kastrinakis and H. Eckelmann, "Measurements of streamwise vorticity fluctuations in a turbulent channel flow," J. Fluid Mech. 137, 165 (1983).

${ }^{6}$ E. G. Kastrinakis, H. Eckelmann, and W. W. Willmarth, "Influence of the flow velocity on a Kovasznay-type vorticity probe," Rev. Sci. Instrum. 50, 759 (1979)

${ }^{7}$ E. G. Kastrinakis, J. M. Wallace, W. W. Willmarth, B. Ghorashi, and R. S. Brodkey, "On the mechanism of bounded turbulent shear flows," Structure and Mechanisms of Turbulence I, Lecture Notes in Physics, Vol. 75 (Springer, Berlin, 1977).

${ }^{8} \mathrm{P}$. Vukoslavčević and J. M. Wallace, "Influence of velocity gradients on measurements of velocity and streamwise vorticity with hot-wire X-array probes," Rev. Sci. Instrum. 52, 869 (1981).

${ }^{9}$ J. F. Foss, "Advanced techniques for transverse vorticity measurements," in Proceedings of the 7th Symposium on Turbulence (University of Missouri-Rolla, Rolla, 1981).

${ }^{10}$ J. F. Foss, S. K. Ali, and R. C. Haw, "A critical analysis of transverse vorticity measurements in a large plane shear layer," Advances in Turbulence (Springer, Berlin, 1987).

${ }^{11}$ J. F. Foss, C. L. Klewicki, and P. J. Disimile, "Transverse vorticity measurements using an array of four hot-wires," NASA CR 178098 (1986).
${ }^{12}$ R. C. Haw, J. K. Foss, and J. F. Foss, "The vortical properties of the high speed region in a plane shear layer and its parent boundary layer," in Advances in Turbulence (Springer, Berlin, 1989).

${ }^{13}$ H. Eckelmann, S. G. Nychas, R. S. Brodkey, and J. M. Wallace, "Vorticity and turbulence production in pattern recognized turbulent flow structures," Phys. Fluids 20, S225 (1977).

${ }^{14}$ J. M. Wallace, "Methods of measuring vorticity in turbulent flows," Exp. Fluids 4, 61 (1986).

${ }^{15}$ J. F. Foss and J. M. Wallace, "The measurement of vorticity in transitional and fully-developed turbulent flows," in Advances in Fluid Mechanics, Lecture Notes in Engineering, Vol. 45 (Springer, Berlin, 1989).

${ }^{16}$ J. M. Wallace and J. F. Foss, "The measurement of vorticity in turbulent flows," Annu. Rev. Fluid Mech. 27, 469 (1995).

${ }^{17}$ J.-H. Kim and H. Fiedler, "Vorticity measurements in a turbulent mixing layer," in Advances in Turbulence, Vol. 2 (Springer, Berlin, 1989).

${ }^{18} \mathrm{P}$. Vukoslavčević, J. M. Wallace, and J.-L. Balint, "The velocity and vorticity vector fields of a turbulent boundary layer. Part I. Simultaneous measurement by hot-wire anemometry," J. Fluid Mech. 228, 25 (1991).

${ }^{19}$ J.-L. Balint, J. M. Wallace, and P. Vukoslavčević, "The velocity and vorticity vector fields of a turbulent boundary layer. Part II. Statistical properties," J. Fluid Mech. 228, 53 (1991).

${ }^{20}$ E. Kit, A. Tsinober, J.-L. Balint, J. M. Wallace, and E. Levich, "An experimental study of helicity related properties of a turbulent flow past a grid," Phys. Fluids 30, 3323 (1987).

${ }^{21}$ E. Kit, A. Tsinober, M. Teitel, J.-L. Balint, J. M. Wallace, and E. Levich, "Vorticity measurements in turbulent grid flows," Fluid Dyn. Res. 3, 289 (1988).

${ }^{22}$ A. Tsinober, E. Kit, and T. Dracos, "Experimental investigation of the field of velocity gradients in turbulent flows," J. Fluid Mech. 242, 169 (1992).

${ }^{23}$ D. B. Lang, "Laser Doppler velocity and vorticity measurements in turbulent shear layers," Ph.D. thesis, Caltech, Pasadena, California, 1985.

${ }^{24}$ R. J. Adrian, "Multi-point optical measurements of simultaneous vectors in unsteady flow-A review,' Int. J. Heat Fluid Flow 7, 127 (1986).

${ }^{25}$ R. J. Adrian, "Particle-imaging techniques for experimental fluid mechanics," Annu. Rev. Fluid Mech. 23, 261 (1991).

${ }^{26}$ W. Merzkirch, Flow Visualization (Academic, New York, 1987).

${ }^{27}$ W. Lauterborn and A. Vogel, "Modern optical techniques in fluid mechanics," Annu. Rev. Fluid Mech. 12, 223 (1984).

${ }^{28}$ L. Hesselink, "Digital image processing in flow visualization," Annu. Rev. Fluid Mech. 20, 421 (1988).

${ }^{29}$ R. B. Miles and D. M. Nosenchuck, "Three-dimensional quantitative flow diagnostics," in Advances in Fluid Mechanics Measurements, edited by M. Gad-el-Hak, Lecture Notes in Engineering (Springer, Berlin, 1989).

${ }^{30} \mathrm{M}$. Gad-el-Hak, Advances in Fluid Mechanics Measurements, Lecture Notes in Engineering (Springer, Berlin, 1989).

${ }^{31}$ F. P. Chiang and G. T. Reid, Optics and Lasers in Engineering, Vol. 9 (Elsevier, New York, 1988).

${ }^{32}$ K. Nishino, N. Kasagi, and M. Hirata, "Three-dimensional particle tracking velocimetry based on automated digital image processing,"' J. Fluids Eng. 111, 384 (1989).

${ }^{33}$ D. Papantoniou and H.-G. Maas, "Recent advances in 3-D particle tracking velocimetry," in Proceedings of the 5th Symposium on Applications of Laser Techniques to Fluid Mechanics (Instituto Superior Técnico, Lisbon, 1990).

${ }^{34} \mathrm{G}$. Haussmann and W. Lauterborn, "Determination of size and position of fast moving gas bubbles in liquids by 3-D image processing of hologram reconstructions," Appl. Opt. 19, 3529 (1980).

${ }^{35} \mathrm{P}$. H. Malyak and B. J. Thompson, "Particle displacement and velocity measurement using holography," Opt. Eng. 23, 567 (1984).

${ }^{36} \mathrm{H}$. Meng and F. Hussain, "Instantaneous flow field in an unstable vortex ring measured by holographic particle velocimetry," Phys. Fluids 7, 9 (1995).

${ }^{37}$ J. Scherer and L. P. Bernal, "Resolution characteristics of holographic particle image velocimetry," AIAA J. 31, 434 (1993).

${ }^{38}$ C.-C. Chu, C.-T. Wang, C.-C. Chang, R.-Y. Chang, and W.-T. Chang, "Head-on collision of two coaxial vortex rings: Experiment and computation," J. Fluid Mech. 296, 39 (1995).

${ }^{39}$ R. E. Falco and C. C. Chu, "Measurement of two-dimensional fluid dynamics quantities using a photochromic grid tracing technique, Photomechanics and Speckle Metrology (SPIE, Bellingham, 1987).

${ }^{40}$ C. Hassa, P. H. Paul, and R. K. Hanson, "Laser induced fluorescence modulation techniques for velocity measurements in gas flows,' Exp. Fluids 5, 240 (1987). 
${ }^{41}$ R. B. Miles, J. J. Connors, E. C. Markovitz, P. F. Howard, and G. J. Roth, "Instantaneous profiles and turbulence statistics of supersonic free shear layers by Raman excitation plus laser induced fluorescence (RELIEF) velocity tagging of oxygen,' Exp. Fluids 8, 17 (1989).

${ }^{42}$ R. B. Miles, D. Zhou, B. Zhang, W. R. Lempert, and Z.-S. She, "Fundamental turbulence measurements by RELIEF flow tagging,' AIAA J. 31, 447 (1993).

${ }^{43}$ A.J. Pearlstein and B. N. Carpenter, "On the determination of solenoidal or compressible velocity fields from measurements of passive or reactive scalars,'’ Phys. Fluids 7, 754 (1995).

${ }^{44}$ A. W. Gruen, "Adaptive least squares correlation: A powerful image matching techniques,' S. Afr. J. Photogram. Remote Sensing, and Chartography 14, 187 (1985).

${ }^{45}$ H.-G. Maas, "Determination of velocity fields in flow tomography sequences by 3-D least squares matching," Proc. 2nd Conf. on Optical 3-D Measurement Techniques, Zurich, 1993 (unpublished).

${ }^{46}$ P. T. Tokumaru and P. E. Dimotakis, "Image correlation velocimetry,", Exp. Fluids 19, 1 (1995).

${ }^{47}$ W. J. A. Dahm, L. K. Su, and K. B. Southerland, "Scalar imaging velocimetry studies of turbulent flow field structure and dynamics,' Bull. Am. Phys. Soc. 36, 2674 (1991).

${ }^{48}$ W. J. A. Dahm, L. K. Su, and K. B. Southerland, "A scalar imaging velocimetry technique for fully resolved four-dimensional vector velocity field measurements in turbulent flows,', Phys. Fluids A 4, 2191 (1992).

${ }^{49}$ W. J. A. Dahm, K. B. Southerland, and K. A. Buch, "Direct, highresolution, four-dimensional measurements of the fine scale structure of Sc $\gg 1$ molecular mixing in turbulent flows," Phys. Fluids A 3, 1115 (1991).

${ }^{50}$ K. B. Southerland, "A A four-dimensional experimental study of conserved scalar mixing in turbulent flows. Ph.D. thesis, The University of Michigan, Ann Arbor, Michigan, 1994.

${ }^{51}$ K. B. Southerland and W. J. A. Dahm, A four-dimensional experimental study of conserved scalar mixing in turbulent flows. Report No. 02677912, Department of Aerospace Engineering (The University of Michigan, Ann Arbor, MI, 1994); submitted to J. Fluid Mech.

${ }^{52}$ L. K. Su and W. J. A. Dahm, "Scalar imaging velocimetry measurements of the velocity gradient tensor field in turbulent flows. II. Experimental results,', Phys. Fluids 8, 1883 (1996).
${ }^{53}$ K. B. Southerland, J. R. Porter, W. J. A. Dahm, and K. A. Buch, “An experimental study of the molecular mixing process in an axisymmetric laminar vortex ring,"' Phys. Fluids A 3, 1585 (1991).

${ }^{54} \mathrm{C}$. Truesdell, The Kinematics of Vorticity (Indiana University, Bloomington, 1954).

${ }^{55}$ A. N. Tikhonov and V. Y. Arsenin, Solutions of Ill-Posed Problems (Winston, Washington, D.C., 1977).

${ }^{56} \mathrm{~K}$. A. Buch, "Fine scale structure of conserved scalar mixing in turbulent shear flows: $\mathrm{Sc} \gg 1, \mathrm{Sc} \approx 1$ and implications for reacting flows," Ph.D. thesis, The University of Michigan, Ann Arbor, Michigan, 1991.

${ }^{57}$ K. A. Buch and W. J. A. Dahm, "Fine scale structure of conserved scalar mixing in turbulent shear flows: $\mathrm{Sc} \gg 1, \mathrm{Sc} \approx 1$ and implications for reacting flows,', Report No. 026779-5, Department of Aerospace Engineering (The University of Michigan, Ann Arbor, MI, 1991).

${ }^{58}$ S. C. Eisenstat, H. C. Elman, and M. H. Schultz, "Variational iterative methods for nonsymmetric systems of linear equations,' SIAM J. Numer. Anal. 20, 345 (1983).

${ }^{59} \mathrm{C}$. Fox, An Introduction to the Calculus of Variations (Dover, New York, 1963).

${ }^{60}$ W. E. Mell, G. Kosály, and J. J. Riley, “The length scale dependence of scalar mixing,', Phys. Fluids A 3, 2474 (1991).

${ }^{61}$ W. E. Mell, V. Nilsen, G. Kosály, and J. J. Riley, “Direct numerical simulation investigation of the conditional moment closure model for nonpremixed turbulent reacting flows,' Comb. Sci. Tech. 91, 179 (1992).

${ }^{62}$ W. E. Mell, V. Nilsen, G. Kosály, and J. J. Riley, “'Investigation of closure models for nonpremixed turbulent reacting flows,' Phys. Fluids 6, 1331 (1994).

${ }^{63}$ L. K. Su, "Scalar imaging velocimetry: Development and application in measurements of fine structure and dynamics in turbulent flows," Ph.D thesis, The University of Michigan, Ann Arbor, Michigan, 1995.

${ }^{64}$ L. K. Su and W. J. A. Dahm, "Scalar imaging velocimetry and its application in measurements of the structure and dynamics of the complete velocity gradient tensor in turbulent flows,' Report No. 026779-14, Department of Aerospace Engineering (The University of Michigan, Ann Arbor, MI, 1995). 